\title{
Pediatric Nasopharyngeal Rhabdomyosarcoma: About a Case and Review of Literature
}

\author{
Dohoué Patricia Eliane Agbanglanon', Imane M'barki', Oswald Houessou1, Gaël Kietga1,

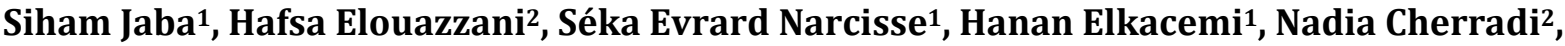 \\ Sanaa Elmajjaoui ${ }^{1}$, Noureddine Benjaafar ${ }^{1}$
}

${ }^{1}$ Department of Radiotherapy Oncology, National Institute of Oncology, Rabat, Morocco

${ }^{2}$ Department of Pathological Anatomy Laboratory, Specialties Hospital, Rabat, Morocco

Email: patriciaagbanglanon@yahoo.fr

How to cite this paper: Agbanglanon, D.P.E., M’barki, I., Houessou, O., Kietga, G., Jaba, S., Elouazzani, H., Narcisse, S.E., Elkacemi, H., Cherradi, N., Elmajjaoui, S. and Benjaafar, N. (2021) Pediatric Nasopharyngeal Rhabdomyosarcoma: About a Case and Review of Literature. Journal of Cancer Therapy, 12, 10-18.

https://doi.org/10.4236/jct.2021.121002

Received: November 11, 2020

Accepted: January 11, 2021

Published: January 14, 2021

Copyright $\odot 2021$ by author(s) and Scientific Research Publishing Inc. This work is licensed under the Creative Commons Attribution International License (CC BY 4.0).

http://creativecommons.org/licenses/by/4.0/

\begin{abstract}
Introduction: Rhabdomyosarcoma (RMS) is the most common soft tissue tumor in children, with the head and neck location accounting for up to $40 \%$ of cases. The embryonal and alveolar histologic variants are more commonly seen in pediatric patients. The treatment is multimodal and the prognosis of this clinical entity is always gloomy. We report the case of nasopharyngeal rhabdomyosarcoma in a 7-year-old boy child with a good response to early post-therapy. Case Presentation: The patient was diagnosed with nasopharyngeal RMS revealed by a congested nose, ptosis and bilateral blindness, and who received induction chemotherapy followed by concurrent radiotherapy followed by adjuvant chemotherapy. The evolution is marked by a good clinical course but persistence of bilateral blindness. Conclusion: The RMS nasopharyngeal often presents with nonspecific symptoms. Multimodal therapy should be performed including surgery, chemotherapy and radiotherapy.
\end{abstract}

\section{Keywords}

Rhabdomyosarcoma, Nasopharyngeal, Pediatric, Chemotherapy,

Radiotherapy

\section{Introduction}

Rhabdomyosarcoma (RMS) is a highly malignant soft tissue tumor that originates from embryonic mesenchymal cells [1]. It is the most common soft tissue tumor in children, with the head and neck region accounting for 35\% - 40\% of cases and is particularly common among younger children [2]. Para meningeal 
(PM) RMSs are tumors that arise from sites adjacent to the meninges, including nasopharynx, nasal cavity, paranasal sinuses, temporal bone, pterygopalatine fossa, and the infratemporal fossa. They have a propensity for central nervous system (CNS) extension and poorer survival than the other RMS. Nasopharyngeal RMSs tends to grow rapidly and invades adjacent structures [2]. The Intergroup Rhabdomyosarcoma Studies (IRSs) and European Studies have established that the ideal management of this para meningeal disease is multimodal, using a combination of surgery, chemotherapy and radiotherapy. We present a case of nasopharyngeal RMS in a seven years old child who was treated by induction chemotherapy followed by radiotherapy then adjuvant chemotherapy.

\section{Case Review}

A 7 years old, boy with no significant past medical history, admitted to our institution, National Institut of Oncology, who presents severe headache that started 8 months ago, accompanied by a congested nose, ptosis and bilateral blindness. He did not have dysphagia or odynophagia, or swallowing, or chewing problems. Neurological examination disclosed third cranial nerve palsy, bilateral ptosis without palpable lymphadenopathy. Computed tomography (CT) imaging showed a tumor of tissular density taking up both nasal cavities and the nasopharynx, measuring $53 \times 33 \times 49 \mathrm{~mm}$. This tumor process invading parapharyngeal spaces, the maxillary sinuses, the pterygoid processes, prevertebral and retrostyloid spaces. It extending into the orbital fissures. It lyses the petrous apex, the clivus and the sphenoidal bone without intracranial extension (Figure 1). Biopsy of the nasopharyngeal process was performed, and the pathological diagnosis was embryonic RMS (Figure 2). Immunohistochemistry (IHC) staining revealed strong positivity for desmin and myogenin (Figure 3 ). The final diagnosis was embryonic RMS. A myelogram and an osteo-medullary biopsy were performed reveals an absence of cells or metastatic clusters. Work-up including chest and abdomen CT scan and skeletal scintigraphy was normal. The patient received 5 cycles of neo-adjuvant chemotherapy bases IVA (Ifosfamide, Vincristine, Actinomycine D). The first CT scan of assessment showed a partial response (Figure 4). Adjuvant radiotherapy treatment is delivered to the planning target volume (PTV).

The treatment plan was performed with intensity modulated radiotherapy (IMRT). Patient was simulated in the supine position, with hyperextension of the neck and the use of a long 5 points thermoplastic mask extending to the shoulder region for immobilization. We performed a contrast-enhanced CT-scan from vertex to C7-T1 level with $3 \mathrm{~mm}$ slights thickness.

All radiotherapy volumes will be at least initially targeting the extent of disease prior to chemotherapy.

The Gross Tumor Volume (GTV) excludes the volume that extends into the normal tissue or cavity, and must include all infiltrative disease detected at initial presentation. 

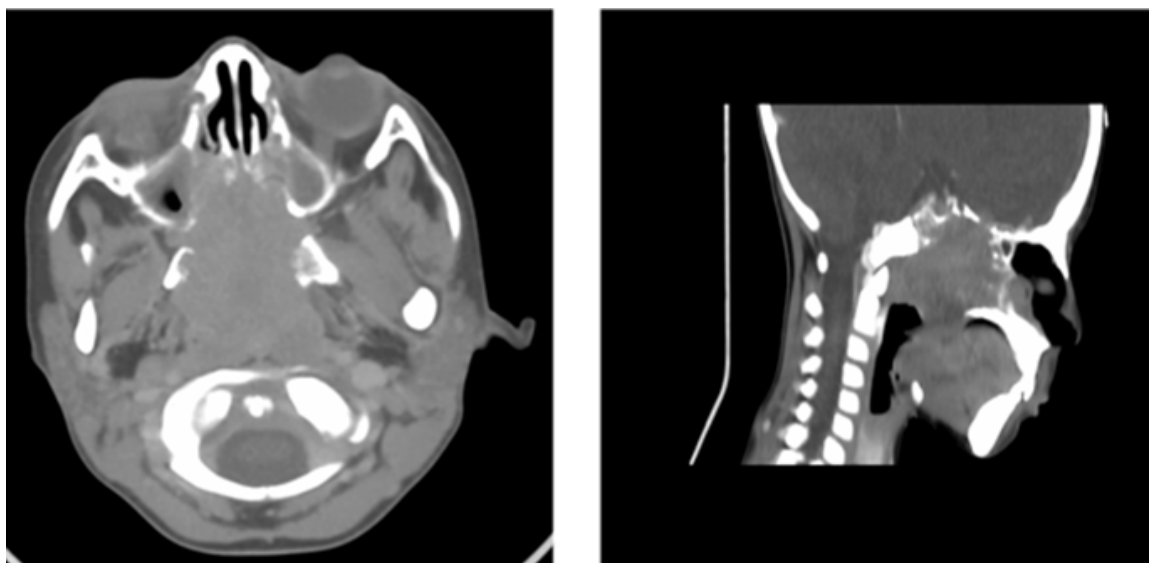

Figure 1. CT-scan image (axial and sagittal) showing the tumor process invading the base of skull.

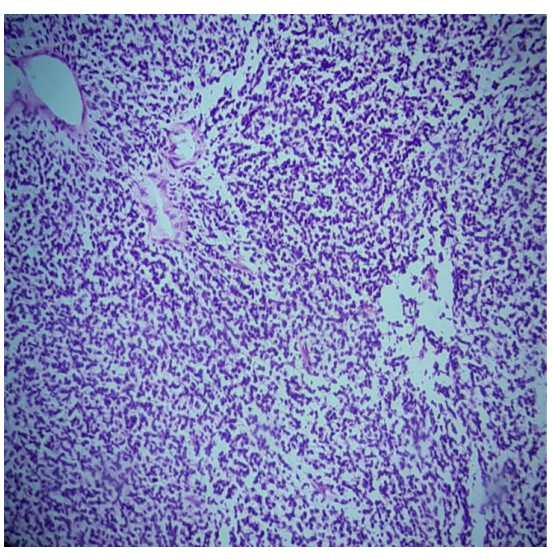

(a)

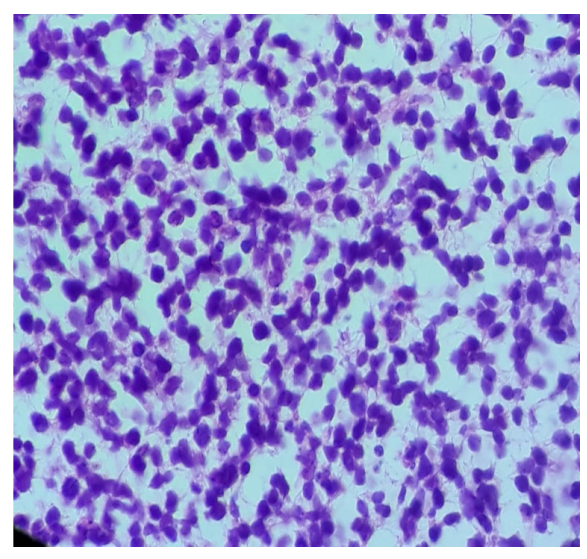

(b)

Figure 2. Histological Hematoxylin-Eosin (HE) staining image, (xG10) (a) and (xG40) (b).

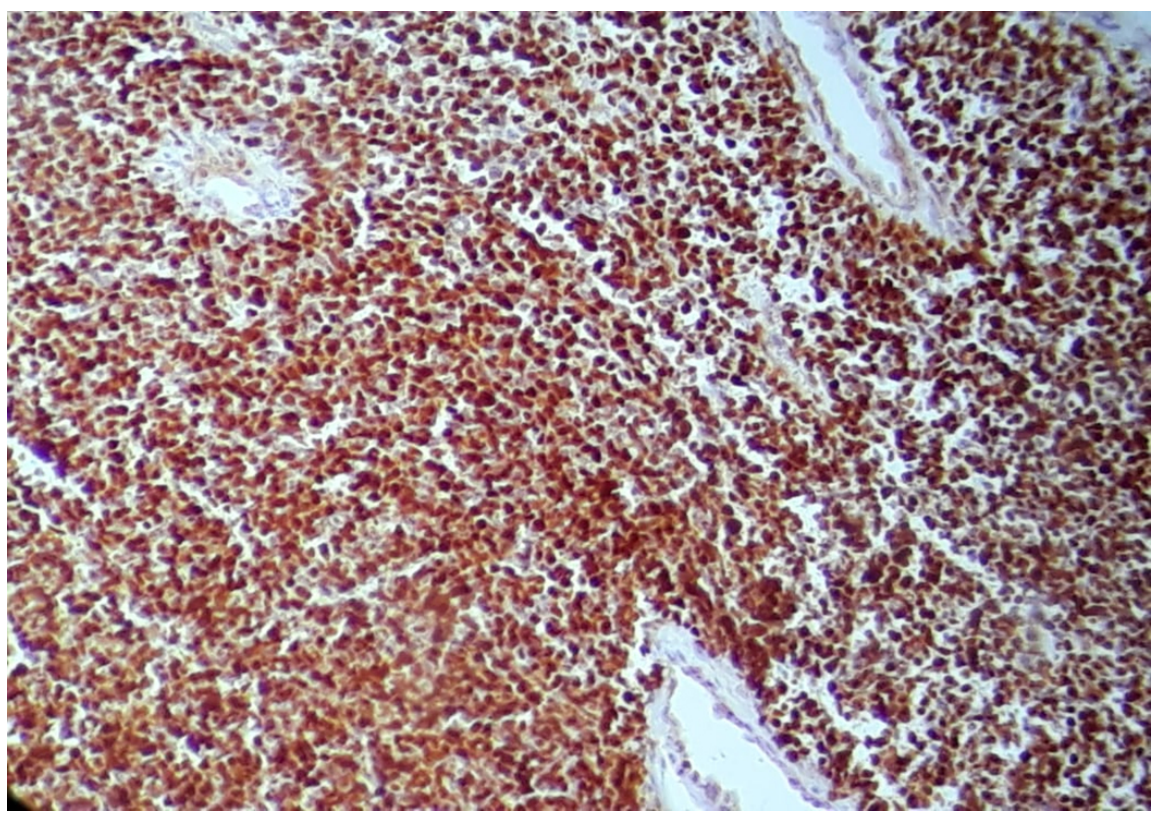

Figure 3. Immunohistochemistry image showing positivity for myogenic antibody. 

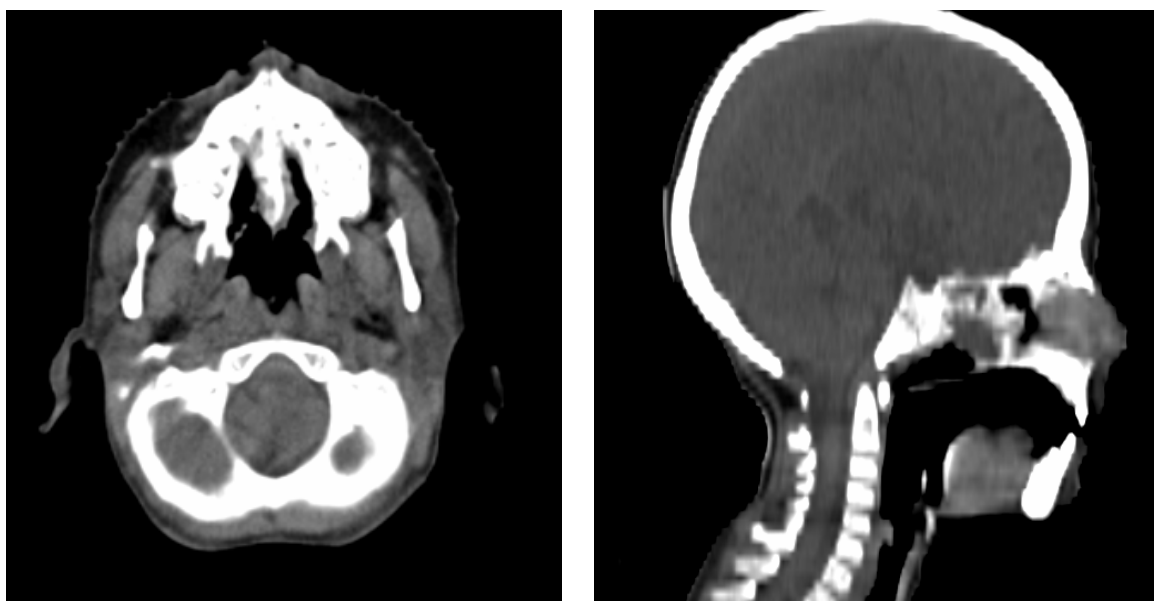

Figure 4. CT-scan image (axial and sagittal) after chemotherapy.

The Clinical Target Volume (CTV) was defined as GTV + a $1 \mathrm{~cm}$ margin according to current Children's Oncology Group (COG) protocols with respected to anatomic barriers. The CTV margins were reduced near critic organs. A 0.5 $\mathrm{cm}$ margin in all directions was added to the CTV to obtain the planning target volume (PTV). Lymph nodes are only included if they are pathologically involved.

The total dose of radiotherapy was 50.4 Gy keeping with the treatment used for Group III tumors (includes para meningeal tumors) according to IRS-IV in 28 fractions of 1.8 Gy per fraction, 5 days per week in five and a half weeks. Relevant organs at risk included the spinal cord, optical chiasma, retina, lens, optic nerves, cochlea, brainstem, hypothalamus and pituitary gland, temporal lobes, oral cavity, parotid and submandibular glands, temporomandibular joint, esophagus, and thyroid gland. Dose constraints to OAR were set according to the RECORD.

He received adjuvant chemotherapy regimen IVA.

Our patient did not exhibit toxicity to chemotherapy but a grade 1 radiodermatitis to radiotherapy.

Parents of the child go through intense moments of stress for the future of their child. The care was provided by social care.

The immediate course is marked by a good clinical course but persistence of bilateral blindness.

\section{Discussion}

Rhabdomyosarcoma (RMS) is a soft tissue tumor which accounts for $4 \%-8 \%$ of all soft tissue tumors in children mostly occurring between the ages of 3 and 12 years [3] [4]. RMS can arise in a variety of anatomic sites throughout the body. The most common primary tumor sites include the head and neck region (35\%) [5] and is particularly common among younger children However. The presentation in the nasopharyngeal is very rare only a few cases have been reported in the literature [6]. The epidemiology of primary tumor presentation is dependent 
upon the histologic variant and age.

The period of time between the onset of symptoms and diagnosis varied about eight months; this delay in diagnosis may be due to the close similarity of the presenting symptoms with those of adenoidal hypertrophy, common in this age group. Signs depend primarily on the location of the tumor. Early symptoms are usually not obvious, showing only nasal congestion, runny nose, nausea, headache and other discomforts. Nasal block, rhinorrhea and recurring otitis media are the most common presenting symptoms [7]. Rapidly progressive eye proptosis is a common manifestation in tumors of the orbit [8]. When local symptoms or lumps appear, the disease is already advanced.

Subtypes of RMS include embryonal (with botryoid variant), alveolar and pleomorphic subtypes according to World Health Organization and Intergroup Rhabdomyosarcoma Studies Committee (IRS). With botryoid variant embryonal form accounts $60 \%-66 \%$ of all [9]. Younger children have embryonal form dominancy whereas adolescents have alveolar type mostly [10]. In our case it was an embryonal rhabdomyosarcoma. Embryonal RMS (ERMS) occurs mostly in the head and neck, retroperitoneum and urogenital system, and rarely in the nasal cavity and sinuses [11].

Physical examination of the head and neck region includes visualization and palpation of the face, scalp, oral cavity and pharyngeal structures, anterior and posterior rhinoscopy, evaluation of cranial nerve function, eye and lymph node examination. When malignancy is suspected, radiological evaluation and biopsy should be performed as quickly as possible [6].

$\mathrm{CT}$ shows the extent of disease and its relation with vital structures. Bone remodeling suggests benign or slow-growing tumors, while bone destruction and loss of soft tissue suggest malignancies. MRI provides superior soft tissue resolution; fat suppression around the facial sinuses, the pterygopalatine fossa and the infratemporal fossa increase the sensitivity of this method for tumor extension. MRI also differentiates the tumor, muscles, secretions, and mucous thickening better, and is superior when assessing perineural and perivascular structures, and intracranial invasion [12].

Considering the fact that $15 \%$ of children with RMS already have distant metastases at the moment of diagnosis [13], it is essential to complete the diagnosis with chest radiograph and/or CT, skeletal scintigraphy. In our case, work-up including chest and abdomen CT scan and skeletal scintigraphy was normal.

Fibrosarcoma, mucormycosis, nasal polyposis and juvenile nasal angiofibroma were considered in the differential diagnosis. The high population count of small round cells makes it imperative to keep Ewing's sarcoma and lymphoma as other important histopathological differentials. The confirmation of the diagnosis is made by immunocytochemistry [10].

RMS histological examination usually demonstrates small round cells with high cytological variability. In the case of poorly differentiated or undifferentiated, immunohistochemical staining is needed to further confirm the diagno- 
sis. Its immunohistochemical markers mainly include myoglobin, myogenic determinant 1 (MyoD1), and skeletal myosin (desmin), muscle-specific actin, myogenin. Negative staining with cytokeratin, S-100 and epithelial membrane antigen helps to exclude the other differential diagnoses for RMS [14].

With the development of radiotherapy and chemotherapy, molecular targeting, gene targeted therapy, and the progress made in the treatment of this disease, at present, the treatment of ERMS multidisciplinary comprehensive treatment methods such as surgery, chemotherapy, radiotherapy, and targeted therapy are mostly used.

Because of the complexity of nasal anatomy and the easy transfer of RMS of the nasal cavity and sinuses into the skull, radical surgery is very difficult [15]. There are classified as clinical group III according to Intergroup Rhabdomyosarcoma Study (IRS) group classification [16].

The surgical management of patients with RMS is site-specific, and attempts for complete tumor resection should be made. Complete tumor resection is generally not advised if there is a significant risk for functional or cosmetic morbidity (for example, in head and neck RMS) [17].

Our case was recused from the surgery and received induction chemotherapy in addition to radiotherapy with adjuvant chemotherapy.

For COG treatment protocols, the three-drug combination of vincristine, actinomycin D, and cyclophosphamide (VAC) has been the backbone of RMS therapy. For certain groups with low-risk disease, there has been interest in treating with only vincristine and actinomycin D to avoid large cumulative alkylator exposure that has been linked to secondary malignancies and sterility, but the results of a recent study found that the local control rates using actinomycin $\mathrm{D}$ were slightly inferior to historical published local control rates using VAC [18]. The greatest need for improvement is in patients with high-risk disease. Recent COG studies have employed the use of a more intensive chemotherapy backbone using vincristine, doxorubicin, and cyclophosphamide that is alternated with ifosfamide and etoposide. Since the survival outlook for high-risk patients has been historically so poor, it appears that a plateau of efficacy has been reached with traditional cytotoxic chemotherapy.

In our case the regimen of chemotherapy was IVA. There was no observed toxicity under this chemotherapy protocol.

The choice of chemotherapy regimen and the duration of treatment are closely related to the improvement of patient survival. The dose of the drug should be based on the patient's condition, age, and tolerance. Applicable as appropriate. For patients with distant metastases, there are reports in the literature that increasing the dose does not improve the patient's prognosis [19].

In the case of parameningeal RMS, there are data to suggest that earlier use of RT may improve local control rates [20].

Reduction of RT volumes has been used if the tumor size decreases after induction chemotherapy. However, in the subset of patients with parameningeal 
RMS and intracranial tumor extension, early RT initiation and wider margin RT to cover adjacent areas at high risk for meningeal extension may be more important for adequate disease control [21].

The preferred radiotherapy technique for pediatric RMS has been sourced from the children's oncology Group (USA): trials ARST 0331, ARST 0431, ARST 0531. Lymph nodes are only included if they are pathologically involved. This technique advises radiotherapy doses of $50.4 \mathrm{~Gy}$ in 28 fractions at $1.8 \mathrm{~Gy}$ per fraction to the isocenter, using $6 \mathrm{MV}$ photons and CT scan planning. Treatment should be once a day, 5 days a week in five and half weeks.

The radiation field normally covers the nasopharynx and both sides of the neck. Accelerators are often used for radiotherapy, and the dose depends on age. The total dose is generally $45-60 \mathrm{~Gy}$, and the course of treatment is 5 - 6 weeks [22].

Such conformal techniques with three dimensional radiotherapy and inverse modulated radiation therapy may help reduce the late effects related to radiotherapeutic management of this tumor [23].

In our case, grade 1 radiodermatitis was observed after $50.4 \mathrm{~Gy}$

Prognostic factors for RMS arising in the head and neck include anatomical site, tumor size, invasiveness, metastatic spread tumor histology and tumor stage. The patients with parameningeal primary tumors in the nasopharynx have been reported to have less favorable survival rates because of the high risk of tumor spreading to the central nervous system [24].

\section{Conclusion}

In summary, encouraging progress in the diagnosis, management and salvage therapy of patients with nasopharyngeal RMS has been made. Chemotherapy and radiotherapy has been highlighted as an effective advance in tumor management. Thus, long-term surveillance is required so that early intervention can be applied.

\section{Conflicts of Interest}

The authors declare no conflicts of interest regarding the publication of this paper.

\section{References}

[1] Gu, M., Yin, W.B., Yu, Z.H., et al. (2009) Radiation Oncology. 4th Edition, China Union Medical College Press.

[2] Healy, J.N., Borg, M.F. (2010) Paediatric Nasopharyngeal Rhabdomyosarcoma: A Case Series and Literature Review. Journal of Medical Imaging and Radiation Oncology, 54, 388-394. https://doi.org/10.1111/j.1754-9485.2010.02187.x

[3] Singh, G.B., Arora, R., Kumar, D., Jain, M., Puri, V. (2013) A Rare Case of Congenital Rhabdomyosarcoma with Review of the Literature. Case Reports in Otolaryngology, 2013, Article ID: 518952. https://doi.org/10.1155/2013/518952

[4] Damor, A., Patel, B., Mapshekhar, R. and Patel, N. (2012) Congenital Rhabdomyo- 
sarcoma: A Rare Axillary Presentation. International Journal of Biomedical and Advance Research, 3, 212-214.

[5] Pappo, A.S. (1994) Rhabdomyosarcoma and Other Soft Tissue Sarcomas of Childhood. Current Opinion in Oncology, 6, 397-402.

https://doi.org/10.1097/00001622-199407000-00012

[6] Iziki, O., et al. (2020) Paediatric Nasopharyngeal Rhabdomyosarcoma: A Rare Case and Literature Review. EC Clinical and Medical Case Reports, 3, 1-5.

[7] Ferman, S.E. (2005) Análise de Sobrevida de Pacientes Pediátricos Portadores de Rabdomiossarcoma: 18 Anos de Experiência do Instituto Nacional de Câncer-RJ. Tese Apresentada à Faculdade de Medicina da Universidade de São Paulo, São Paulo.

[8] Walterhouse, D. and Watson, A. (2007) Optimal Management Strategies for Rhabdomyosarcoma in Children. Pediatric Drugs, 9, 391-400. https://doi.org/10.2165/00148581-200709060-00006

[9] Mondal, P.K., Pal, I., Misra, S., Biswas, S., Bera, S.P. (2009) Rhabdomyosarcoma of Nose, Nasopharynx and Paranasal Sinuses. Indian Journal of Otolaryngology and Head \& Neck Surgery, 61, 317-319. https://doi.org/10.1007/s12070-009-0090-9

[10] Hicks, J. and Flaitz, C. (2002) Rhabdomyosarcoma of the Head and Neck in Children. Oral Oncology, 38, 450-459. https://doi.org/10.1016/S1368-8375(01)00105-1

[11] Yu, X.P., Liang, Z.Y. and Wang, P. (2006) Imaging Manifestations of Rhabdomyosarcoma in Adults. Journal of Clinical Radiology, 25, 612-615.

[12] Burkat, C.N. and Lucarelli, M.J. (2005) Rhabdomyosarcoma Masquerading as Acute Dacryocystitis. Ophthalmic Plastic \& Reconstructive Surgery, 21, 456-458.

https://doi.org/10.1097/01.iop.0000181349.79287.12

[13] Crist, W., Gehan, E.A., Ragab, A.H., Dickman, P.S., Donaldson, S.S., Fryer, C., Hammond, D., Hays, D.M., Herrmann, J., Heyn, R., et al. (1995) The Third Intergroup Rhabdomyosarcoma Study. Journal of Clinical Oncology, 13, 610-630. https://doi.org/10.1200/JCO.1995.13.3.610

[14] Kumar, S., Perlman, E., Harris, C.A., Raffeld, M. and Tsokos, M. (2000) Myogenin Is a Specific Marker for Rhabdomyosarcoma: An Immunohistochemical Study in Paraffin-Embedded Tissues. Modern Pathology, 13, 988-993. https://doi.org/10.1038/modpathol.3880179

[15] Guo, L.M., Zhao, H., Chi, F.L. and Yan, Y.H. (2001) Clinical Analysis of 17 Cases of Rhabdomyosarcoma in Ear Nose and Throat. Journal of Clinical Otorhinolaryngology, 15, 358-359 . http://dx.chinadoi.cn/10.3969/j.issn.1001-1781.2001.08.010

[16] Daya, H., Chan, H.S., Sirkin, W. and Forte, V. (2000) Pediatric Rhabdomyosarcoma of the Head and Neck: Is There a Place for Surgical Management? Archives of Otolaryngology_ Head and Neck Surgery, 126, 468-472.

[17] Leaphart, C. and Rodeberg, D. (2007) Pediatric Surgical Oncology: Management of Rhabdomyosarcoma. Surgical Oncology, 16, 173-185.

https://doi.org/10.1016/j.suronc.2007.07.003

[18] Raney, R.B., Walterhouse, D.O., Meza, J.L., Andrassy, R.J., Breneman, J.C., Crist, W.M., Maurer, H.M., Meyer, W.H., Parham, D.M. and Anderson, J.R. (2011) Results of the Intergroup Rhabdomyosarcoma Study Group D9602 Protocol, Using Vincristine and Dactinomycin with or without Cyclophosphamide and Radiation Therapy, for Newly Diagnosed Patients with Low-Risk Embryonal Rhabdomyosarcoma: A Report from the Soft Tissue Sarcoma Committee of the Children's Oncology Group. Journal of Clinical Oncology, 29, 1312-1318. https://doi.org/10.1200/JCO.2010.30.4469 
[19] Skubitz, K.M. and D’Adamo, D.R. (2007) Sarcoma. Mayo Clinic Proceedings, 82, 1409-1432. https://doi.org/10.4065/82.11.1409

[20] Arndt, C.A., Hawkins, D.S., Meyer, W.H., Sencer, S.F., Neglia, J.P. and Anderson, J.R. (2008) Comparison of Results of a Pilot Study of Alternating Vincristine/Doxorubicin/Cyclophosphamide and Etoposide/Ifosfamide with IRS-IV in Intermediate Risk Rhabdomyosarcoma: A Report from the Children's Oncology Group. Pediatric Blood \& Cancer, 50, 33-36. https://doi.org/10.1002/pbc.21093

[21] Eaton, B.R., McDonald, M.W., Kim, S., Marcus Jr., R.B., Sutter, A.L., Chen, Z. and Esiashvili, N. (2013) Radiation Therapy Target Volume Reduction in Pediatric Rhabdomyosarcoma: Implications for Patterns of Disease Recurrence and Overall Survival. Cancer, 119, 1578-1585. https://doi.org/10.1002/cncr.27934

[22] Wu, X., Li, P., Xie, L., Zhang, X.Y., Wang, W., Xiao, J.H. and Wang, F. (2008) Clinical Analysis of 13 Cases of Embryonal Rhabdomyosarcoma in the Nose. Journal of Clinical Otorhinolaryngology Head and Neck Surgery, 22, 338-341. http://dx.chinadoi.cn/10.3969/j.issn.1001-1781.2008.08.002

[23] Kraus, D.H., Saenz, N.C., Gollamudi, S., Heller, G., Moustakis, M., Gardiner, S., Gerald, W.L., Ghavimi, F. and La Quaglia, M.P. (1997) Pediatric Rhabdomyosarcoma of the Head and Neck. American Journal of Surgery, 174, 556-560. https://doi.org/10.1016/S0002-9610(97)00171-2

[24] Weiss, S.W. and Goldblum, J.R. (Eds.) (2001) Rhabdomyosarcoma. Enzinger and Weiss's Soft Tissue Tumors. 4th Edition. CV Mosby, St. Louis, 785-835. 